\title{
THE EFFECT OF FOOTWEAR TO THE POSTURE
}

\author{
Marianna Trebuňová, Mária Danko, Lucia Bednarčíková, Monika Michalíková, \\ Jozef Živčák
}

\author{
Department of Biomedical Engineering and Measurement, Faculty of Mechanical Engineering, \\ Technical University of Kosice, Kosice, Slovakia
}

\begin{abstract}
The aim of this study is to assess the effect of footwear on postural status of a group of volunteers representing the general population of female students. Based on the assumption that the elegant shoe with tapered toe and high heel does not provide adequate foot support, the study was designed to assess its direct link and impact to body segment alignment and resulting negative effect on posture. Repetition of this misalignment ensures the individual circumstances of posture. Due to the heel elevation, the weight is transferred to the distal part, resulting in a postural response of the entire musculoskeletal system to maintain balance. The group consisted of 30 women of the age 18-28 years with an average age of 22.7 years, height $167 \pm 0.3 \mathrm{~cm}$ and weight $57 \pm 0.9 \mathrm{~kg}$. Anamnesis and aspection were performed to assess of the occurrence of shortened and weakened muscles and subsequently the patient's standing was examined. Two static methods were chosen for evaluation of the posture. The first one was the Silhouette Posture Analysis and the evaluation method by Jaros and Lomnicka. The result was the identification of typical muscle imbalance as the most common presumption of faulty posture, and it was confirmed that footwear affects a person's natural posture. Differences in the sensitivity of the two methods were also identified and studies are not only appropriate that but also evaluate differences in impact among subjects.
\end{abstract}

\section{Keywords}

high heels, flat shoes, postural stability, posture, posturology, Silhouette Posture Analysis

\section{Introduction}

Body posture is defined as the alignment of body segments which is considered as an important health indicator. Normal human posture is the characteristic of the vertical position which relies on spinal alignment and its position over the patient's head and pelvis [1].

The word posture comes from the Latin "ponore" which means to place it. It is an active posture controlled by the CNS according to an anatomically defined movement system while respecting biomechanical principles, muscular activity, against the effects of external and internal forces. The posture reflects and affects the overall condition of the human organism and encompasses all the motor skills of a human with the aim of maintaining a certain position, alignment [2, 3]. Posture describes the orientation of a given segment relative to a force vector. Posture is not determined only when standing on two legs, but e.g. even when sitting or standing or on one leg [2, 4, 5]. The muscles actuate the postural function. Thus, the body requires their interplay within certain muscle groups to maintain balance in the gravitational field. Gravity, as well as external or internal forces acting on the body, have a great influence on maintaining an upright posture. Internal forces include muscle strength, ligament strength, and bone pressure to the bones [2, 5, 6].

The concept of the upright posture requires the interplay of individual muscle groups within the superficial and deep musculature with the participation of the passive component of the bone, cartilage, and ligamentous apparatus under the control of the CNS [7]. In addition to the above, the psyche significantly contributes to stabilization [2, 5, 8]. It is necessary to start from the personality of the person and consider upright posture as an individual postural program, which originated during the movement development of the individual, formed from birth throughout life and changes as a dynamic phenomenon depending on external and internal conditions. Everyone has his or her individual posture as an expression of somatic and mental personality [2]. The individual posture is influenced and shaped by endogenous factors of biological origin. This means the current state of health and genetic predisposing factors, overcoming accidents, diseases, congenital or acquired deformities, etiology and others. Furthermore, there are exogenous factors such as unilateral load, insufficient or excessive physi- 
cal activity, prolonged sitting and standing, inappropriate shoes, permanent tension, and long-term stress, etc. Combination of several factors can lead to severe posture disorders [2, 3, 7, 8].

The issue of footwear is one of the favourite topics of conversation in the public. This is interesting for each person who uses shoes every day, not to mention the fashion. The appearance of a person is very important for business, and therefore people have always been looking for elegant shoes with a narrow toe. Literature showed that wearing high heels can produce injurious effects on several structures from the toes to the pelvis $[9,10]$. No studies considered shoe length as an impacting factor on walking with high heels [11]. The reaction of the organism to unsatisfactory conditions is the emergence of some functional and organic disorders. And it is precisely functional changes occurring based on muscle imbalance, in which there are no objective pathological findings in terms of structural defects, it is necessary to diagnose, treat and optimally prevent them in a timely manner [2, 11, 12].

\section{Influence of shoe type on posture}

The results of many studies declare that wearing high-heeled shoes, as well as a narrow shoe, cause improper body posture, rounding or flattening of the spinal curve, sides, protruding shoulder blades, flat chest, incorrect pelvis alignment, asymmetric shoulder height, muscle imbalance and limitation of the range of movement, etc., see Fig. 1 [9, 10, 13-16].

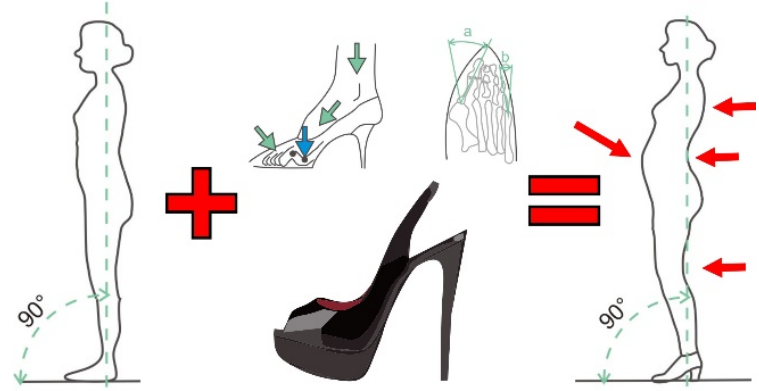

Fig. 1: The effect of shoes on posture.

Wearing high heels leads to various foot disorders and injuries such as metatarsal pain, Achilles tendon tension, plantar fasciitis and Haglund malformation [12-14, 17]. However, there is little available information explaining the specific mechanisms and reasons why wearing high heels causes foot deformity [17]. Footwear with heel higher than $\leq 5 \mathrm{~cm}$ creates a continuous pressure on the forefoot, worsening leg deformities such as hallux valgus, hammer fingers, causes blistering, etc. Clinical studies [9, 10, 12] confirm that longterm wearing of such shoes leads to an increase in the size of the lordosis and the shortening of the Achilles tendon, as well as other diseases and problems. Increasing the heel height decreases the ankle's range of movement and increases the forefoot contact with the pad. High heel also restricts movement in the ankle, knee, and hip joint. The ankle joint is almost immobilized. Wide and low heel maximizes stability and minimizes tension on metatarsal heads. Heel height affects the mechanics of the foot, the entire lower limb, and the spine. Higher heels relieve leg and plantar flexors, but the muscles of the lower limbs and the muscles of the pelvis and torso are more stressed. Also, the step is shortened, pelvic rotation occurs, and hip flexion is increased, leading to increased lumbar lordosis. The study showed that walking in shoes with heels $\geq 10 \mathrm{~cm}$ significantly increases cervical muscle activity in healthy women. Walking with high heels requires more metabolic energy than low shoes. Changing body segments also changes the overall centre of gravity of the body. When walking with high heels, the centre of gravity of the body has been shown to be higher in the vertical direction [9, 12-14].

Wang et al. investigated the foot kinematics of high heel wearers and compare any differences with barefoot individuals using the Oxford Foot Model (OFM). Their reference group were fifteen healthy women aged 20-25 years were measured while walking barefoot and when in high heels. Compared to the barefoot, participants wearing high heels demonstrated larger hallux dorsiflexion, and less hallux plantarflexion during the initial stance phase. There was also greater forefoot adduction. The hind foot demonstrated a larger dorsiflexion in the horizontal plane, greater internal rotation, and decreased peak hind foot extension rotation. These findings complement existing kinematic evidence that wearing high heels can lead to foot deformities and injuries [17].

Sipio et al. considering not only the heel height but also the foot/shoe size in a sample of 21 healthy women in three different walking conditions: 1) barefoot, 2) wearing $12 \mathrm{~cm}$ high heel shoes independently from shoe size, and 3) wearing shoes with heel height based on shoe size, keeping the ankles' plantar flexion angle constant. Comparing the three walking conditions showed significant differences between both high heeled conditions (variable and constant height) and barefoot in spatio-temporal, kinematic and kinetic parameters. Regardless of the shoe size, both heeled conditions presented a similar gait pattern and were responsible for negative effects on walking parameters [11].

The aim of our study was to confirm the effect of wearing high heel shoes on the posture and to determine differences of two posture analysis methods for identification the impact of different footwear.

\section{Methods}

The study was conducted at the Department of Biomedical Engineering and Measurement, Technical University of Kosice and each participant gave written 
informed consent to the recording procedure. The study procedures involved non risk to participants therefore research ethics committee approval was not necessary.

First, each study participant went through a detailed medical history, a questionnaire and a basic asymmetry examination by aspection, evaluation of the overall posture as well as the alignment of separate body parts $[2-4,18]$. The group consisted of 30 women of the age 18-28 years.

Data for the Jaros and Lomnicka and the evaluation by Silhouette Posture Analysis, were assessed based on photos. These were taken perpendicular to the frontal and sagittal plane to avoid distortion of the results. The field of view represented the top of the head to the sole of the foot. The pictures were taken of the overall posture from a distance $150 \mathrm{~cm}$ from the subject. Separate anatomy, such as head and neck, chest, chest to knee and entire lower limbs were photographed from a distance $80 \mathrm{~cm}$ from the subject $[3,4,18]$.

Basic measured position is upright position, head up, chin level is parallel to the floor, neck pulled up, chin with neck at $90^{\circ}$ angle, chest up, lower abdomen flat and shoulders relaxed. The arms hang easily with the hand resting near the hip, the palms facing the body and the elbows slightly bent so that the forearms hang slightly forward, thumbs pointing forward. Shoulder blades retracted back and down, pelvic muscles naturally toned, feet parallel, toes evenly spaced on the pad, lower limbs axis straight $[3,4,18]$.

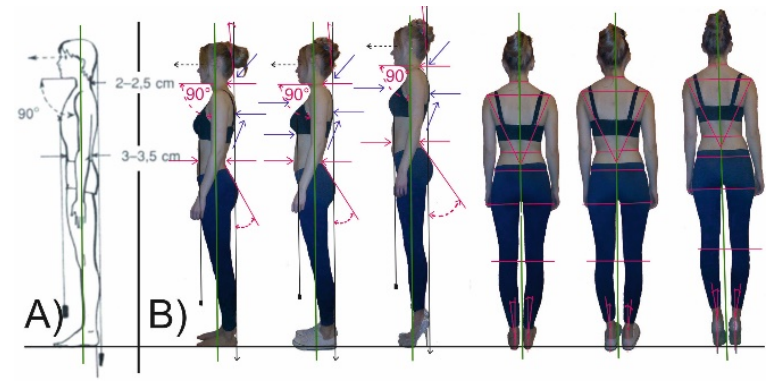

Fig. 2: Standing-body alignment A) Lateral View-Good posture. B) Lateral View and Posterior View Posture.

Ideal vertical alignment is viewed from the side, the plumb line passes through the earlobe or centre of the ear, the centre of the shoulder (the acromion process) if the arms are hanging in normal alignment, either through (or just behind) the cervical and lumbar vertebrae and slightly in front of the bodies of the thoracic vertebrae, slightly behind (posterior to) the centre (axis) of the hip joint (from the side view, the greater trochanter), slightly in front (anterior) of the knee joint, slightly in front (anterior) of the ankle joint (the lateral malleolus) [3, 4, 18].

\section{Posture according to Jaros and Lomnicka}

The posture evaluation according to Jaros and Lomnicka is focused on 6 indicators of posture. That is holding the head and neck, the thorax curvature angle, abdomen with pelvis inclination, back curve, posterior view, and lower limbs (valgus and varus deformities of knees and ankles).

Individual body segments are graded 1-4, with 1 being the norm. Posture is evaluated by the sum of points from the measured segments, where a score of up to 5 points represents a perfect posture, 6-10 points represents a good posture, 11-15 points represents a poor posture and 16-20 points indicates a very poor posture.

The lower limb values are not included in the postural stability evaluation. These values are considered in the overall posture evaluation. The obtained marks are written in a fraction, in the numerator the sum of the scoring areas 1 to 5 and in the denominator is the mark for the lower limbs, e.g. Fig. 2 shows volunteer number 6 with barefoot evaluating, flat shoes, high heels shoes (6:6:7), so overall with good rating. According to Jaros and Lomnicka, the scoring of the posture also includes the evaluation of the lower limbs, which, however, is not included in the overall evaluation of posture and is quoted in a fraction of the denominator position $[3,18]$.

\section{The Silhouette Postural Analysis}

The processing and evaluation of the photos by the Silhouette Postural Analysis is rated based on 12 indicators. In the frontal plane, the position of head is evaluated (the perpendicular passes through the highest point on the head and through the vertebra C7, the horizontal passes through the acromion), the shoulders are scored (the perpendicular passes through the highest point on the head and through the vertebra C7, the horizontal passes through the acromions), the spine is evaluated (the vertical line passes through C7 and the seat line, the change in the spine curve is considered), the position of the hips is rated (the vertical line went through the seat line and the horizontal line went through the narrowed part of the hips) [3, 4, 19].

In the sagittal plane the position of the cervical vertebrae (deviation from the vertical, posture of the neck in front of the vertical indicates posture of the body as very bad), the thorax curvature angle based (shape monitoring and possible structural deformity of the anterior thoracic wall) and holding of the arms (shape monitoring from the top and scapula inclination) are rated. In the field of evaluation of the torso, abdomen and the lumbar spine, the vertical line is pulled over the shoulder joint and trochanter, and mainly the change of position towards lordosis and the abdominal is monitored. When scoring the thoracic part of the spine, the change in thoracic kyphosis versus the vertical is evaluated. The position of the legs is rated based on a vertical stretch between the lower limbs and the symmetrical position of the ankles relative to that vertical is assessed. Finally, condition of foot is evaluated [3, 4, 19]. 
Each segment is graded by mark 1,2,3. Category A is considered the standard, which represents a perfect posture and thus 12 points. Category B with 13-24 points represents good posture and category $\mathrm{C}$ represents faulty posture and the individual achieved a total of 25-36 points [3, 4, 19].

We determined that 30 subjects should be included based on the reported experience of others. Comparisons between rating were conducted with Shapiro-Wilk Test and Kruskal-Wallis Test with $\mathrm{p}<0.05$ considered statistically significant.

\section{Results}

The group consisted of 30 women of the age 18-28 years, see Table 1 . Based on the case history of respondents interviewed, $30 \%$ of them had lower limb injuries in the last 10 years. Eight of the women surveyed reported a fracture or sprain of the ankle, two of them fractures of the toes and three of them reported a torn knee ligament. Disease affecting posture was reported in $23 \%$ of the observed (the most often due to mild scoliosis or hyper-lordosis or due to flat feet). 30\% reported leg pain and 59\% reported spinal pain that was not caused by injury or disease.

Insoles for shoes were used by $30 \%$ of respondents. One volunteer occasionally used gel pads with heeled shoes, 5 subjects said they were buying mass-produced walking shoe insoles for comfort while walking, 3 respondents regularly wore individual insoles prescribed by a doctor to their heeled shoes. The most common causes were flat feet, lower limbs pain when walking or standing for extended periods. Less common reasons were comfort or pain in the plantar fascia. The average height heel shoes were $10 \mathrm{~cm}$. Barefoot shoes are not used by any survey participant. Heeled shoes and flat shoes ranged in sizes 38-41.

During the photo-shooting, none of the respondents had insole in their shoes. Six respondents had a shaped arch in shoes without heels, while no respondent had a shaped arch in the high heeled shoes.

Table 1: Participants.

\begin{tabular}{|ccc|}
\hline $\begin{array}{c}30 \\
\text { participants }\end{array}$ & Spread & Mean STD \\
\hline Age & $18-28$ years & 22.7 years \\
Height & $156-178 \mathrm{~cm}$ & $167 \pm 0.3 \mathrm{~cm}$ \\
Weight & $46-85 \mathrm{~kg}$ & $57 \pm 0.9 \mathrm{~kg}$ \\
\hline \multicolumn{2}{|c|}{ Preferred shoes } & Number of \\
\hline Flat shoes $\leq 2.5 \mathrm{~cm}$ & respondents \\
Heel shoes $<5 \mathrm{~cm}$ & 9 \\
High heel shoes $\geq 5 \mathrm{~cm}$ & 7 \\
\hline
\end{tabular}

\section{Evaluation according Jaros and Lomnicka}

Average values of research subjects $(n=30)$ in each of 3 categories (barefoot, flat, high heels) of evaluated posture ranged around 10 score.

According to rating Jaros and Lomnicka, without evaluated score of lower extremities, we found that $63.3 \%$ of barefoot data reflected good rating, $36.7 \%$ poor rating. Data from flat shoes showed different in 5 cases of study simples so $46.7 \%$ has good rating and $53.3 \%$ has poor. The results of posture without evaluated score of lower extremities on high heels determined that $53.3 \%$ had good rating and $46.7 \%$ had poor rating, so a change in categorization occurred in 3 cases, with reference values barefoot.

The posture of the lower limbs changed most often. In heeled shoes, $20 \%$ of subjects improved their overall foot position compared to the lower extremities of the barefoot. On the contrary, in $36.6 \%$ the position deteriorated. The second area with the most frequent change was the evaluation of the abdomen and pelvic inclination. In $16.7 \%$ of cases, pelvic and abdominal posture improved in high heeled shoes and worsened in $20 \%$ compared to barefoot measurement. When evaluating the head and neck area, $6.7 \%$ of subjects improved their position and $26.7 \%$ volunteers increased anteflexion in high heeled shoes. The least numerous were changes in posture in the frontal plane. In this assessment, $6.6 \%$ of subjects improved their posture and in $3.3 \%$ of volunteers worsened body posture in high heeled shoes.

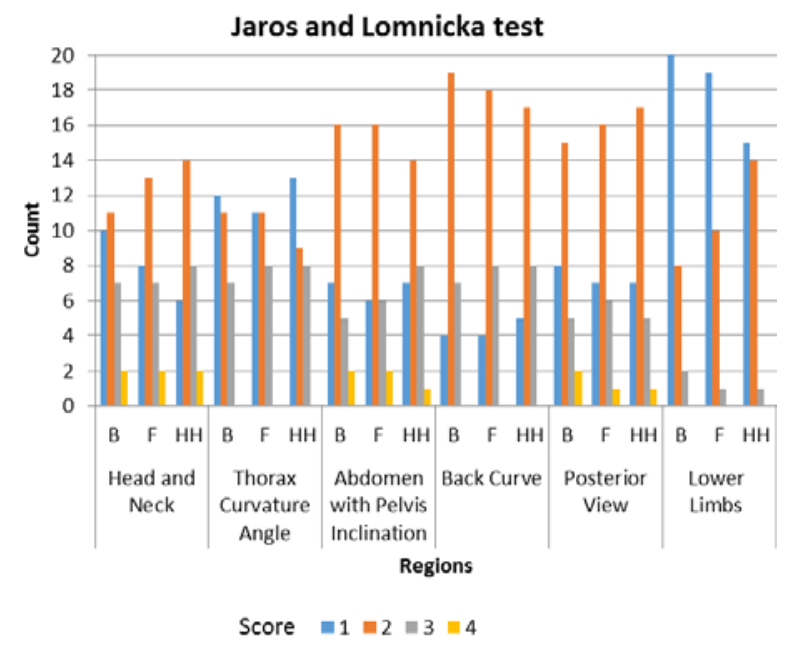

Fig. 3: The count of score of individual body segments according rating of Jaros and Lomnicka (B-Barefoot, F - Flat Shoes, $\mathrm{HH}$ - High heels).

Based on the measured data, a change of body posture in both the sagittal and frontal plane at $73.3 \%$, which represents 22 respondents was observed. Of this, posture worsened in $43.3 \%$ of the sample of female 
students, and in $20.0 \%$ of cases abdominal posture and pelvic tilt improved, reducing both lordosis and the position of lower limbs relative to standing on heeled shoes of about $10 \mathrm{~cm}$, while the baseline data were from barefoot rating. The change in posture in flat shoes was seen in $36.7 \%$ of which $10 \%$ of people had changed their posture in flat shoes, while in the heeled shoes they had the same rating as barefoot. The remaining $26.7 \%$ of the respondents did not change their body posture.

The measured and evaluated data show that the monitored subjects have a perfect posture in the area of Lower limbs, however these are worsening with highheeled shoes. The lowest count of very good postures is in the Back Curve area and the largest number of poor postures is in the Thorax Curvature Angle area. The count of the posture with the high heels compared to the barefoot occurred in the region of Head and Neck, where there was also a relatively high part of the poor posture, see Fig. 3.

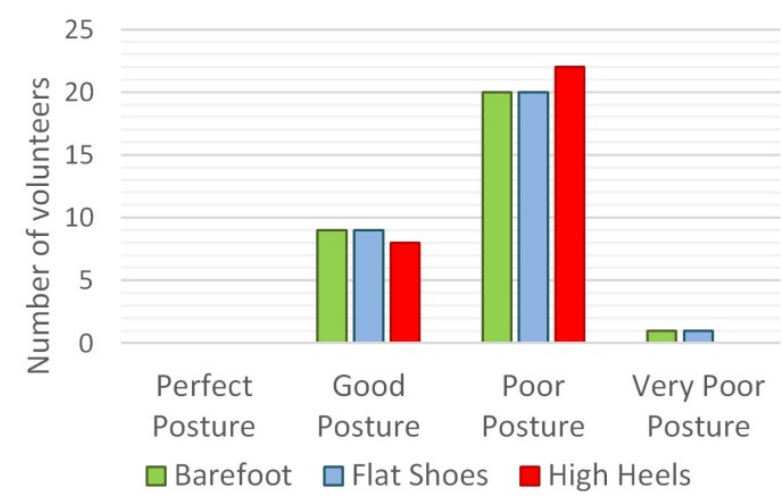

Fig. 4: The overall posture evaluation in volunteers according to posture quality.

According to the overall posture evaluation we found that $30 \%$ of the volunteers have good posture barefoot and in flat shoes, but in high heels it was $26.7 \%$. Poor posture was found in $66.7 \%$ barefoot and in flat shoes, in high heels it was 73.3\%. Posture quality barefoot and on wearing flat shoes was in 1 case $(3.3 \%)$ very poor, which means round back, rounded, and drops shoulders, tilt pelvis and flat foot, see Fig. 4.

The distribution of the data was determined by the Shapiro-Wilkow test, which showed that there was not normal distribution. Therefore, the nonparametric Kruskal-Wallis test was used for further statistical data processing. All different proportions were not statistically significant $(\mathrm{p}<0.05$ considered statistically significant).

\section{The Silhouette Posture Analysis}

This analysis found all belong to the category of good posture, based on the 12 indicators in all 3 conditions examined (barefoot, flat shoes, high heels).

The highest values of rating were recorded in shoulder elevation, lumbar spine, and lateral pelvic tilt. The greatest deviations from the barefoot reference values were found in the shoulder's alignment in the frontal plane, in neck anteflexed, lateral pelvic tilt and lower limbs.
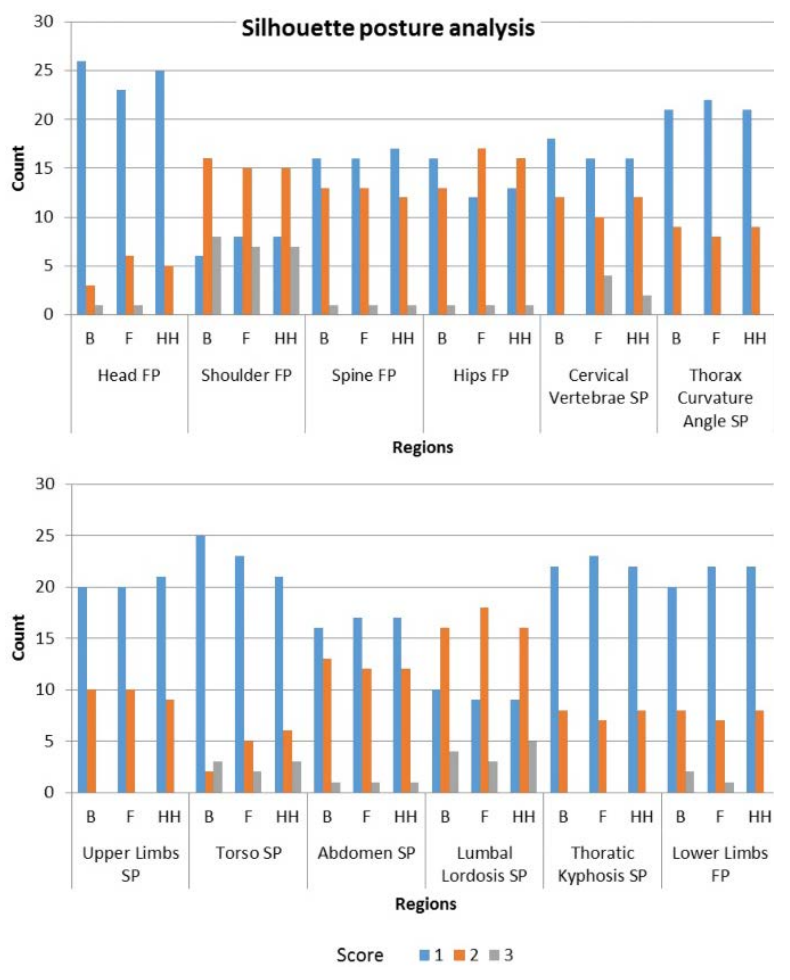

Fig. 5: Count of score from the Silhouette Postural Analysis of 12 body alignment segments (B-Barefoot, $F$ - Flat Shoes, HH - High heels).

The position of hips and legs changed most often (see Fig. 5). In $23.3 \%$ of subjects had a deterioration in hip heeling in high shoes and improved in $13.3 \%$. When evaluating the legs, in $23.3 \%$ of the respondents improved the position of the legs and $13.3 \%$ worsened when putting on the heeled shoes. Another most prominent area is the lateral view evaluation of the neck, where neck holding worsened in $20 \%$ of sample of female students and improved in $6.6 \%$ of this ensemble. A change in head posture was seen in $13.3 \%$ of subjects for the better and $13.3 \%$ of subjects for the worse. The remaining evaluations did not show such numerous changes in posture.

The Silhouette Posture Analysis showed a change in posture in $63.3 \%$ of the measured subjects, while in $26.7 \%$ the posture improved and in 30\% evaluated posture worsened in heeled shoes. In $3.3 \%$ of subjects, body posture in non-heeled shoes changed, while in heeled shoes they had the same rating as barefoot.

The measured and evaluated data show that according to the Silhouette Posture Analysis, the highest number of perfect posture is in Head and Trunk regions, good posture in Shoulders and Lumbar regions. Faulty posture is the most represented in Shoulder region, see Fig. 5. 
The distribution of the data was determined by the Shapiro-Wilkow test, which showed that there was not normal distribution. Therefore, the nonparametric Kruskal-Wallis test was used for further statistical data processing. All different proportions were not statistically significant $(\mathrm{p}<0.05$ considered statistically significant).

\section{Discussion}

We used two standard methods to evaluated 30 women student volunteers to determine the impact of type of shoes on posture. We demonstrated that the type of footwear appears to impact posture, but that the magnitude of the impact varied between subjects. We also found that Jaros and Lomnicka method appears to be more sensitive to the changes.

The presented study was focused on the evaluation of young women; therefore, the age of subjects was limited from 18 to 30 years. Effects might be different in older woman. A group of 30 subjects participated in the measurement, according to the results it would be appropriate to expand the group to at least 50-100 subjects, which follows from similar studies [1, 9, 14, $15,20]$. A sample size power calculation based on a specific hypothesis should be conducted to determine the appropriate number of subjects before proceeding.

In addition, it can be stated that due to footwear significant corrections in alignment can be achieved, but also can cause deformities or development of chronic musculoskeletal disorders accompanied by algic symptomatology (pain) [20, 21]. It appears that the global trend barefoot shoes could be an interesting focus point of prevention and at the same time an important treatment procedure for common corrections e.g. when dealing with hallux, toe deformity, flat foot, etc., $[9,14,15,17]$ as its basic feature is a spacious toe, thin, straight and maximum flexible sole providing enough irritant information to proprioceptors on the sole of the foot. The result is a foot reaction leading to stimulation of the respective central nervous structures responsible for the postural stability of the axial organ that is dominant in posture.

The evaluation according to Jaros and Lomnicka uses 4-level score in 6 regions, the Silhouette Posture Analysis uses 3-level score in 12 regions. It follows that the sensitivity of the evaluation is higher for the first method. The output of the evaluation of the 12 regions should provide a more comprehensive picture of the posture, but the individual regions affect each other when the second method is used, which is most pronounced in the cases-Abdomen vs. Lumbal Lordosis, Torso Thoratic Kyphosis vs. Upper limbs, Torso Thorax Curvature Angle.
The count of perfect postures in the individual regions does not match according to the two methods used (the Jaros and Lomnicka test-lower limbs, the Silhouette Posture Analysis-Head).

Based on two visual methods, we observed a modification postural stability when changing the type of footwear in 73.3\% according to Jaros and Lomnicka and in $63.3 \%$ by using Silhouette Postural Analysis of all the 30 subjects tested. Of this, according to Jaros and Lomnicka posture worsened in $43 \%$ of the sample, and in $20 \%$ of cases abdominal posture and pelvic tilt improved, reducing both lordosis and the position of lower limbs relative to standing on heeled shoes of about $10 \mathrm{~cm}$, while the baseline data were from barefoot rating. While in $26.7 \%$ study samples according Silhouette Postural Analysis, the posture improved and in $30 \%$ evaluated worsened in heeled shoes.

According to rating Jaros and Lomnicka of barefoot data was found that $56.7 \%$ of the samples has good posture and by the Silhouette Postural Analysis has good posture $100 \%$ of study samples.

Both visual methods show that height heel affects head posture, deepens anteflexion, shoulder asymmetry, back curve and the entire lower limb. The high heels relieved the lower limbs flexors, but muscles of the lower limbs, pelvis and torso are more stressed [5, 17, 20].

The most striking deviations of posture according Jaros a Lomnicka were reported in anteflexion degree of the head and in the position of the ankle joint and knee. Due to the different soles and heels of the shoes, the valgus stance improved and the position of the varusive lower extremities deteriorated, where e.g. flatfooted subjects occurred to a visually positive change in the high-heeled shoes. Effect the high heeled shoes on varus feet in leads to the misalignment of the feet, the offset from the media axis increases.

By the Silhouette Postural Analysis were found the most significant deviations occurred in the evaluation of the shoulders and lumbar spine, where was differences in the acromion symmetry, which is most often caused by uneven shoulder load or muscle imbalance due to a combination of factors such as consequence of uneven load e.g. handbags, muscle imbalance, inactivity or a sedentary lifestyle. Perhaps this method is more sensitive to these kind of postural changes.

In the clinical practice is a more widespread method of the posture evaluation Method according Jaros and Lomnicka than Silhouette Postural Analysis. The second one is more complex in the terms of measurement and evaluation (time comsumption, higher number of operations, risk of errors). The evaluation by both methods is highly subjective, so it is appropriate that it be performed by the same person when used in clinical practice. 


\section{Conclusion}

The key finding of this study is that the final score appears not sufficient for a comprehensive evaluation of the posture for both methods, however it is necessary to take into account the evaluations in individual regions and different subjects.

The results suggest that it would be necessary and appropriate to continue the study with a larger number of subjects. It would also be appropriate to investigate the view of clinical practice to the practical application of both methods. The presented topic is highly current due to the confirmed impact of shoe selection to the health of the all population.

\section{Acknowledgement}

The work has been supported by research grants: Stimulus for Research and Development of Ministry of Education No. 1233/2018, Cultural and educational grant agency of the Ministry of Education, Science, Research and Sports of the Slovak Republic: KEGA 041TUKE-4/2019, KEGA 023TUKE-4/2020 and Research and Development Support Agency APVV19-0290.

\section{References}

[1] Stolinski L, Kozinoga M, Czaprowski D, Tyrakowski M, Cerny $\mathrm{P}$, Suzuki N, et al. Two-dimensional digital photography for child body posture evaluation: Standardized technique, reliable parameters and normative data for age 7-10 years. Scoliosis and Spinal Disord. BioMed Central Ltd. 2017;12:1-24.

[2] Molnárová M: Posture, its meaning, diagnostics and disorders. Rehabilitacia [Internet]. 2009 Dec [cited 2020 Jun 15]; 46(4):195-206. Available from: https://www.rehabilitacia.sk/archiv/cisla/4REH2009-m.pdf

[3] Pernicová H, Bělková-Preislerová T, Javůrek J, Kyralová M, Labudová J, Strnad P. Zdravotní tělesná výchova. Praha: Fortuna; 1992. 184 p.

[4] Matascikova S. Vplyv obuvi na posturu [bachelor's thesis]. Košice: TUKE; 2017.

[5] Quin E, Rafferty S, Tomlinson C. Safe Dance Practice, Human Kinetics. Australia: Human Kinetics Australia P/L; 2015.

[6] Kubat O, Ovadia D. Frontal and sagittal imbalance in patients with adolescent idiopathic deformity. Ann Transl Med. 2020 Jan;8(2):29. DOI: 10.21037/atm.2019.10.49

[7] Mansfield PJ, Neumann DA. Essentials of Kinesiology for the Physical Therapist Assistant. 3rd ed. Mosby; 2019. Chapter 8, Structure and Function of the Vertebral Column; p.178-232. DOI: 10.1016/B978-0-323-54498-6.00008-4

[8] Bogduk N. Functional anatomy of the spine. Handb Clin Neurol. 2016;136:675-88. DOI: 10.1016/B978-0-444-53486-6.00032-6

[9] Russell BS, Muhlenkamp KA, Hoiriis KT, Desimone CM. Measurement of lumbar lordosis in static standing posture with and without high-heeled shoes. J Chiropr Med. 2012 Sep;11(3): 145-53. DOI: 10.1016/j.jcm.2012.02.002
[10] Baaklini E, Angst M, Schellenberg F, Hitz M, Schmid S, Tal A, Taylor WR, Lorenzetti S. High-heeled walking decreases lumbar lordosis. Gait Posture. 2017 Jun;55:12-4. DOI: 10.1016/j.gaitpost.2017.03.035

[11] Di Sipio E, Piccinini G, Pecchioli C, Germanotta M, Iacovelli C, Simbolotti C, et al. Walking variations in healthy women wearing high-heeled shoes: Shoe size and heel height effects. Gait Posture [Internet]. 2018;63:195-201. Available from: http://www.sciencedirect.com/science/article/pii/S0966636218 30479X. DOI: 10.1016/j.gaitpost.2018.04.048

[12] Barnish MS, Barnish J. High-heeled shoes and musculoskeletal injuries: a narrative systematic review. BMJ Open. 2016;6: e010053. DOI: 10.1136/bmjopen-2015-010053

[13] Yu J, Wong DW, Zhang H, Luo ZP, Zhang M. The influence of high-heeled shoes on strain and tension force of the anterior talofibular ligament and plantar fascia during balanced standing and walking. Med Eng Phys. 2016 Oct;38(10):1152-6. DOI: 10.1016/j.medengphy.2016.07.009

[14] Michoński J, Witkowski M, Glinkowska B, Sitnik R, Glinkowski W. Decreased Vertical Trunk Inclination Angle and Pelvic Inclination as the Result of Mid-High-Heeled Footwear on Static Posture Parameters in Asymptomatic Young Adult Women. Int. J. Environ. Res. Public Health. 2019 Nov 18; 16(22):4556. DOI: 10.3390/ijerph16224556

[15] Franklin S, Li FX, Grey MJ. Modifications in lower leg muscle activation when walking barefoot or in minimalist shoes across different age-groups. Gait Posture. 2018 Feb;60:1-5. DOI: 10.1016/j.gaitpost.2017.10.027

[16] Živčák J, Bednarčíková L, Michalíková M. Protetika a ortotika - kalceotika. Košice: Technická univerzita v Košiciach; 2010. $202 \mathrm{p}$

[17] Wang M, Gu Y, Baker JS. Analysis of foot kinematics wearing high heels using the Oxford foot model. Technol Health Care. 2018;26(5):815-23. DOI: $10.3233 /$ THC-181264

[18] Vaněčková L. Comparison body-control boys and girls of younger school age [master's thesis]. Brno: KTV PdF MU; 2008.

[19] McRoberts LB, Cloud RM, Black CM. Evaluation of the New York Posture Rating Chart for Assessing Changes in Postural Alignment in a Garment Study. Cloth. Text. Res. J. 2013 Apr; 31(2):81-96. DOI:10.1177/0887302X13480558

[20] Mika A, Oleksy Ł, Kielnar R, Świerczek M. The influence of high- and low-heeled shoes on balance in young women. Acta Bioeng Biomech. 2016;18(3):97-103. DOI: 10.5277/ABB-00483-2015-02

[21] Goud A, Khurana B, Chiodo C, Weissman BN. Women's musculoskeletal foot conditions exacerbated by shoe wear: an imaging perspective. Am J Orthop. 2011 Apr;40(4):183-91.

Doc. RNDr. Marianna Trebuňová, PhD. Department of Biomedical Engineering and Measurement

Faculty of Mechnical Engineering Technical University of Kosice Letna 9, SR-04200 Kosice

E-mail: Marianna.trebunova@tuke.sk Phone: +421556022358 